46 | 2011

Accueil et formation des enfants étrangers en France de la fin du XIX $X^{e}$ siècle au début de la Deuxième Guerre mondiale

\title{
Accueil des enfants juifs étrangers en France et leur sort sous l'Occupation
}

Sabine Zeitoun

\section{(2) OpenEdition \\ Journals}

Édition électronique

URL : https://journals.openedition.org/dhfles/2108

DOI : $10.4000 /$ dhfles.2108

ISSN : 2221-4038

Éditeur

Société Internationale pour l'Histoire du Français Langue Étrangère ou Seconde

Édition imprimée

Date de publication : 1 janvier 2011

Pagination : 123-144

ISSN : 0992-7654

Référence électronique

Sabine Zeitoun, "Accueil des enfants juifs étrangers en France et leur sort sous l'Occupation », Documents pour l'histoire du français langue étrangère ou seconde [En ligne], 46 | 2011, mis en ligne le 25 septembre 2012, consulté le 31 mai 2021. URL : http://journals.openedition.org/dhfles/2108 ; DOI : https://doi.org/10.4000/dhfles.2108

Ce document a été généré automatiquement le 31 mai 2021.

(c) SIHFLES 


\title{
Accueil des enfants juifs étrangers en France et leur sort sous l'Occupation
}

\author{
Sabine Zeitoun
}

$1 \mathrm{Au}$ cours des années trente, par vagues successives, des dizaines de milliers de juifs fuyant l'Allemagne, l'Autriche, la Pologne et la Tchécoslovaquie viennent se réfugier en France. La France est le principal pays d'accueil de ces réfugiés juifs qui représentent $40 \%$ de ceux qui ont quitté l'Allemagne entre 1933 et 1939. Sur les 25000 réfugiés, $85 \%$ sont juifs ${ }^{1}$. Parmi eux, il y a de nombreux enfants et adolescents dont certains arrivent seuls, livrés à eux-mêmes, ne parlant pas ou à peine le français. En 1933, plusieurs associations d'aide se mobilisent pour les accueillir comme le Comité de bienfaisance israélite de Paris (créé en 1809 sous Napoléon), le Comité de défense des juifs persécutés d'Allemagne, le Comité d'aide et d'accueil aux réfugiés allemands victimes de l'antisémitisme. Pour coordonner leurs activités, plusieurs groupes fusionnent pour donner naissance au Comité d'assistance aux réfugiés (CAR) en juillet $1936^{2}$.

\section{Les premières maisons d'enfants de l'OSE ${ }^{3}$ en France}

Dès 1934, un an après l'avènement d'Hitler au pouvoir, l'Union-OSE, organisation juive internationale à vocation médico-sociale, qui avait été fondée en Russie tsariste et qui vient d'ouvrir sa succursale à Paris, crée sa première colonie de vacances à la Varenne en banlieue parisienne $e^{4}$. Riche d'une solide expérience dans le domaine éducatif, l'Union-OSE y accueille, pour un séjour de vacances de quatre à huit semaines, plus d'une centaine d'enfants allemands ou polonais, âgés de 3 à 7 ans, arrivés sans leurs parents. La plupart de ces enfants sont dans un état psychologique déplorable. Certains souffrent d'une grave instabilité nerveuse. Étant très perturbés suite à l'important traumatisme qu'ils ont subi dans leur pays, il leur faut impérativement une prise en charge spécifique qui prenne en compte leur douloureux vécu. 
3 De plus, et alors qu'ils sont en pleine croissance, nombre d'entre eux ont souffert de carences alimentaires à cause des difficultés économiques auxquelles leurs familles ont été confrontées. La doctoresse Françoise Brauner confirme que

sur le plan médical, j'ai constaté une certaine instabilité psychomotrice très sensible des enfants : chez les uns, il s'agissait non seulement d'un chagrin durable, mais de l'expression du dépaysement. Chez d'autres, une certaine sousalimentation, en pleine croissance, se faisait sentir : depuis 1933, bien des familles avaient eu du mal à survivre économiquement. ${ }^{5}$

4 À Paris, un patronage fonctionne comme centre d'observation pour enfants difficiles. Cet établissement pour enfants juifs de 6 à 13 ans, regroupés sans distinction de nationalité, est créé sous la direction du docteur Pollnow, médecin-psychiatre juif allemand, nouvellement réfugié de Berlin. Il élabore un programme médical et pédagogique qui « comprend des jeux, des promenades éducatives, l'enseignement du chant et l'initiation à des travaux manuels ». Les enfants sont surveillés après leur sortie et des conseils sont prodigués aux personnes chargées de leur éducation (en premier lieu les parents) ${ }^{6}$. De plus, «à côté de l'observation du comportement spontané de ces enfants du point de vue intellectuel, affectif, moteur et social, on évalue également leurs capacités mentales ${ }^{7}$.

5 Le nombre de juifs réfugiés s'accroît sensiblement après le déchaî-nement de violence de la « Nuit de Cristal » (Kristallnacht), en novembre 1938. Ils sont des milliers à fuir leur pays pour gagner principalement la France mais aussi les Pays-Bas et la Belgique. Au Royaume-Uni, un mouvement regroupant des associations juives et non juives comme les Quakers ou l'YMCA entreprend une véritable opération de sauvetage. Elle se propose d'accueillir provisoirement en Angleterre dix mille enfants originaires d'Allemagne principalement mais aussi d'Autriche et de régions annexées en Tchécoslovaquie et en Pologne. Les parents de ces enfants sont souvent décédés ou internés dans des camps. Cette opération, intitulée "Kindertransport », c'est-à-dire «transport d'enfants», a pu faire venir presque 10000 enfants. Parti de Berlin, un premier convoi constitué uniquement d'enfants de moins de 17 ans (parmi lesquels quelques bébés) arrive en Grande-Bretagne le $1^{\mathrm{er}}$ décembre 1938, soit moins d'un mois après la Nuit de Cristal. Jusqu'à la déclaration de guerre en septembre 1939, plusieurs trains partent aussi des autres grandes villes d'Allemagne mais égale-ment de Vienne, Dantzig et Prague. Les enfants sont aussitôt dispersés dans des familles d'accueil, des institutions ou des foyers répartis sur l'ensemble du territoire britannique ${ }^{8}$.

6 Parallèlement, en France, des directives administratives durcissent l'entrée et l'installation des nouveaux réfugiés fuyant l'Autriche annexée. Cependant, le Comité israélite pour les enfants venant d'Allemagne et d'Europe centrale, créé en décembre 1938 sous l'autorité du CAR, obtient l'autorisation de faire venir en France des enfants à la condition que leur subsistance leur soit assurée jusqu'à leur majorité.

7 Aussi, ce comité intervient-il par le biais de l'CEuvre de secours aux enfants (OSE), déjà citée. Pour secourir les nouveaux venus, cette dernière ouvre des établissements dotés de personnels éducatifs formés au travail social et originaires d'Europe centrale, tout comme leurs pensionnaires. Cette action est menée sous la conduite du pédagogue autrichien Ernst Papanek, militant socialiste réfugié depuis 1937 en France. L'OSE lui confie la direction générale de ses maisons d'enfants de la région parisienne. Au nombre de quatre, elles ont été ouvertes entre 1933 et 1939. Trois sont dans le secteur de Montmorency ; la quatrième est située à Eaubonne, au château de La Chesnaie. 


\section{Une pédagogie adaptée à la spécificité de l'enfance réfugiée}

8 Le programme pédagogique a comme préalable la « rééducation » de ces enfants. Des enfants effarouchés, piétinés, battus. Il s'agit d'« en refaire des êtres humains ».

Les principes éducatifs en vigueur s'articulent autour de trois axes fondamentaux :

1. la déculpabilisation des enfants par rapport à leur situation de réfugiés,

2. la revalorisation de leur identité juive,

3. le développement de leur esprit combatif afin de faciliter leur intégration.

10 Pour ces enfants, il va s'agir d'adopter une autre langue, une autre culture mais sans toutefois renier leur propre histoire. Ce sont, d'ailleurs, des médecins juifs allemands ayant subi eux-mêmes des persécutions qui s'en occupent.

11 Pour Papanek, l'éducation morale et la formation du caractère de ces jeunes doivent les préparer à devenir des adultes libres et conscients de leurs responsabilités. Il ne faut pas qu'ils ressentent les persécutions subies comme la manifestation d'un sort inéluctable. Il convient au contraire de les armer pour qu'ils luttent avec courage contre toutes les difficultés. Pour ce faire, au sein de ces maisons, des conférences et des discussions sont organisées, ainsi que des représentations théâtrales dont ils sont eux-mêmes les acteurs. Les fêtes juives comme Pessah et Hanoukka sont célébrées et, à ces occasions, des cadeaux leur sont remis ${ }^{9}$. L'école publique joue un rôle important pour ces enfants qui ont été brutalement déscolarisés. $75 \%$ de ceux qui sont en âge scolaire fréquentent l'école communale. Comme les enfants d'Allemagne et d'Autriche sont issus pour la plupart de milieux favorisés, ils disposent d'un bagage culturel et intellectuel plus important et sont souvent à même d'apprendre et de progresser plus rapidement que les autres.

12 En 1939, 123 enfants, âgés de 7 à 14 ans et réfugiés d'Allemagne quelques mois après la Nuit de Cristal,trouvent refuge au château de l'œuuvre de la Guette à Villeneuve-SaintDenis en Seine-et-Marne. Ce manoir appartient à la baronne Edmond de Rothschild. Le départ de ces enfants n'avait été ni prévu ni préparé. La plupart de leurs parents étaient emprisonnés en Allemagne et avaient dû renoncer à leur autorité parentale sans avoir eu le temps d'y réfléchir. C'est à cette condition que leurs enfants avaient pu sortir du territoire. D'autres étaient déjà orphelins. Ils avaient vécu des situations douloureuses et traumatisantes. La situation matérielle et sociale de leurs familles avait basculé suite à l'instauration des lois raciales. Ils avaient subi des humiliations et des brimades de la part de certains de leurs camarades de classe ou de leurs professeurs.

Lotte S., née en 1924 à Berlin, en témoigne :

C'était difficile car partout il y avait des panneaux d'interdiction. Nous ne pouvions nous montrer nulle part : au cinéma, à la plage, sur les bancs des jardins publics. Tout nous était interdit. Sur les vitrines était inscrit de ne pas acheter chez les juifs. Avec la haine attisée par le Stürmer ${ }^{10}$ et la radio, mon assurance avait disparu. Je me sentais diminuée. Les filles allemandes allaient au $B D M^{11}$. Je voulais aussi y participer mais n'étais qu'une "sale juive ». ${ }^{12}$

Léo B., 12 ans en 1938 (né à Hönningen, Palatinat, en 1926) :

[...] Mon père était employé commercial avant que la firme aryenne le licencie. Alors il s'est retrouvé chômeur. Pendant un temps, il a pu toucher une allocation. 
Après quatre mois, il a trouvé du travail dans une quincaillerie comme « astiqueur " de métaux. C'était un travail très dur. Il a eu du travail jusqu'au 10 novembre (1938), puis il a été arrêté... [...] À six heures du matin, la foule est arrivée devant la maison en hurlant «Allez-y! Tuez ces Juifs! Qu'on les jette dans le feu à la synagogue ". ${ }^{13}$

\section{blottissaient entre elles au moment de leur arrivée :}

Assez vite, j'ai compris qu'elles se communiquaient leurs chagrins à propos de leurs familles "abandonnées» en Allemagne. Il ne s'agissait pas de la tristesse qui s'empare de tous les enfants qui arrivent dans une maison d'enfants - quoiqu'elle existât, bien évidemment -, mais de l'angoisse intense d'ignorer le sort de la mère, de la grand-mère, livrées au banditisme nazi, ou du père déjà déporté dans un camp de concentration. ${ }^{15}$

17 Au sein de cette maison, l'encadrement était composé de trois couples d'éducateurs professionnels ou de médecins qui connaissaient bien la culture allemande et sa langue ou étaient eux-mêmes autrichiens ou allemands. À titre d'exemple, le couple Alfred et Françoise Brauner avaient déjà eu une expérience quelque peu similaire auprès d'enfants lors de la guerre d'Espagne. Ils pouvaient exprimer leur traumatisme par le dessin, le graphisme, les couleurs; traumatisme qu'ils ne pouvaient pas exprimer oralement. D'autre part, organisés en "République d'enfants ", selon les méthodes du pédagogue russe Makarenko, les enfants pouvaient prendre en charge leur quotidien et devenir progres-sivement indépendants :

Pour nous, le programme éducatif était clair, et nous étions une fois de plus tous d'accord: consolider l'autonomie des enfants afin de renforcer leur sentiment de sécurité ; leur confier des tâches réelles dans la marche de la maison ; les faire sortir à l'extérieur autant que faire se pouvait. ${ }^{16}$ a été rapide, dans la mesure où ils savaient lire et écrire dans leur pays et pour certains, étaient issus d'un milieu aisé. L'école de la République, pour peu de temps encore, jouera son rôle d'intégration dans la société française. À cet égard, le respect voire une infinie reconnaissance pour le pays d'accueil, la France, étaient partagés par les éducateurs et les enfants. La devise républicaine "Liberté, Égalité, Fraternité » était souvent évoquée.

Par ailleurs, l'Organisation-Reconstruction-Travail (ORT) dispensait à ces enfants un enseignement professionnel comme la couture pour les filles ou la maroquinerie pour les garçons. Dès son origine, l'ORT, organisation philanthropique, née en Russie tsariste à la fin du XIX siècle, s'était consacrée au développement de l'artisanat et de l'agriculture pour sortir les populations juives de la misère en leur permettant d'acquérir une qualification professionnelle. Encore en activité de nos jours, cette organisation internationale est présente en France depuis 1921.

Hors du commun, l'expérience des maisons a duré environ six mois, c'est-à-dire jusqu'à la déclaration de guerre. Évacués à la Bourboule (Puy-de-Dôme) au début du conflit, ces enfants seront confiés à l'OSE à partir de novembre 1941.

21 Par ailleurs, la mobilisation en faveur des enfants réfugiés d'Europe centrale n'échappe pas aux bonnes volontés. Ainsi, le 3 juillet 1939, le Comte Hubert de Monbrison, Secrétaire général du Secours aux enfants de réfugiés politiques, met le château de Quincy-sous-Sénart à la disposition d'un groupe de 40 garçons venus de Berlin. Cette 
demeure avait auparavant hébergé quelques jeunes filles russes venues en 1917 puis, beaucoup plus tard, de jeunes réfugiées espagnoles. Après la débâcle du printemps 1940, une partie des 40 garçons est confiée à l'OSE, comme le sont ceux de l'orphelinat de la Varenne.

En juin 1939, l'arrivée des juifs étrangers s'est effectuée dans des condi-tions parfois quelque peu rocambolesques, comme au moment de l'épo-pée du Saint-Louis. Parti de Hambourg pour La Havane, ce paquebot transportait plus de 900 juifs en provenance d'Allemagne et d'Autriche, dont 200 enfants. Tous avaient des visas leur permettant de se réfugier provisoirement à Cuba. Mais suite à un changement d'orientation de la politique cubaine concernant l'accueil des réfugiés, les passagers ne sont plus autorisés à entrer à Cuba. Le Saint-Louis essaye ensuite vainement de les faire débarquer à Miami. Pendant plus d'un mois, le bateau erre ainsi sur les mers sans pouvoir accoster nulle part et retourne finalement en Europe. Après d'âpres négociations au sujet des conditions de leur prise en charge, notamment entre organisations juives, comités d'entraide et autorités gouvernementales, quatre pays acceptent finale-ment de les accueillir. La Belgique en reçoit 214, les Pays-Bas 181 et la Grande-Bretagne 288. La France en recueille quant à elle 224. Parmi eux, il y a plus d'une cinquantaine d'enfants ${ }^{17}$. L'OSE les ayant pris en charge, ils seront intégrés dans un foyer à Montmorency.

\section{L'OSE face aux camps d'internement de la zone libre}

L'occupation allemande de Paris contraint les dirigeants de l'OSE à évacuer les enfants qui sont à sa charge en zone non occupée, particu-lièrement en Haute-Vienne et dans la Creuse où des châteaux sont aménagés à cet effet. Au fil des mois, d'autres établissements s'ouvrent dans l'Allier, le Var, etc. Ces homes vont être pour les enfants qui y sont regroupés de véritables refuges familiaux; tout du moins jusqu'aux grandes rafles d'août 1942. Ils y mènent une vie quasi normale. Leur scolarité est assurée principalement par les écoles communales. Cepen-dant, un complément d'instruction leur est apporté au sein des homes eux-mêmes. Ce sont surtout des activités ludiques ou culturelles, musicales notamment. Certains y reçoivent aussi une éducation religieuse. Ces tâches sont confiées à un personnel éducatif en majorité d'origine étrangère, donc à même de comprendre la spécificité culturelle et linguistique de ces enfants ou adolescents ${ }^{18}$. Par ailleurs, certains enseignants juifs français, exclus de la fonction publique et donc interdits d'enseignement par le Statut des juifs du 3 octobre 1940, mettent leur savoir au service des pensionnaires de ces homes.

Ces maisons sont principalement créées au cours des deux premières années de l'Occupation, au fur et à mesure que s'accroît le nombre d'enfants pris en charge. Il y en aura plus d'une vingtaine dans le sud de la France.

Pour beaucoup de juifs venus en France pour échapper aux persécutions, le répit a été bref. À la déclaration de guerre, en septembre 1939, Allemands et Autrichiens, considérés comme "ressortissants de pays ennemis", sont enfermés dans des camps insalubres, avec leurs enfants.

Parmi ces camps, figurent entre autres ceux de Poitiers dans la Vienne (route de Limoges), de Gurs dans les Basses-Pyrénées ou encore celui des Milles dans les Bouchesdu-Rhône. Par la suite, une loi, celle du 4 octobre 1940, devait conférer aux préfets le pouvoir d'interner «les étrangers de race juive » dans des camps spéciaux; ceux déjà 
cités et bien d'autres encore. Comme le mentionne à juste titre Geneviève ArmandDreyfus (cf. ici-même), c'est bien sous la Troisième République que le gouvernement Daladier a pris des mesures d'exception pour répondre à l'arrivée massive et illégale des réfugiés espagnols sur le territoire français. Des camps ont été créés dans le sud de la France, le long des Pyrénées. À l'origine, ils ont servi au regroupement des réfugiés républicains espagnols.

L'objectif principal pour la plupart des œuvres caritatives juives et non juives est de pénétrer dans ces camps dans lesquels le gouvernement de Vichy a fait interner à partir de l'automne 1940 des milliers de familles juives étrangères. Les œuvres qui ont l'autorisation d'y pénétrer s'efforcent dans un premier temps de prodiguer des soins et d'améliorer la situation alimentaire. En effet, rien n'avait été prévu pour ces internés. Cependant, très vite, le constat s'impose de lui-même : l'état sanitaire des enfants est particulièrement préoccupant. La moindre des maladies infantiles s'aggrave faute de soins disponibles. La sous-alimentation fait le reste. đEdèmes de la faim, oisiveté, fragilité psychologique, etc. sont le lot commun de beaucoup de ces enfants.

L'urgence devient alors de faire sortir le plus grand nombre d'enfants âgés de moins de 15 ans, en commençant par ceux dont la santé physique et mentale est jugée la plus alarmante, afin de les accueillir dans des maisons d'enfants, en particulier dans celles de l'OSE. Ce n'est qu'après de longues démarches administratives que leur libération peut se faire en toute légalité. On les fait d'abord séjourner au centre de Palavas-lesFlots dans l'Hérault pour leur administrer des soins intensifs et les réalimenter progressivement. Après avoir transité plus ou moins longtemps à Palavas, ils intègrent ensuite les autres homes de l'OSE.

Une fois la santé recouvrée, certains enfants vont ensuite pouvoir émigrer légalement aux États-Unis ou en Amérique latine. Les autres resteront quant à eux dans les pensionnats de l'OSE.

À partir de juin 1942, on y développe progressivement une activité spécifique qui occupera une part sans cesse plus importante dans le dispositif éducatif. Cette activité, c'est le sport. Un jeune juif originaire de Strasbourg, GeorgesLoinger, est chargé d'en développer la pratique. Responsable du mouvement de jeunesse sioniste Hatikva, il est également moniteur national d'éducation physique des EIF, c'est-à-dire des Éclaireurs israélites de France. C'est à ce titre qu'il est promu formateur, sur le plan sportif, des éducateurs des homes. Avec des stages mais aussi par le déclenchement d'alertes fictives, on s'efforce d'entraîner les jeunes à réagir au mieux en cas d'urgence : se préparer en quelques minutes, emporter avec soi le strict minimum, courir dans les bois, se dissimuler, s'échapper instinctivement tout en maîtrisant sa peur, etc.

31 Par la suite, Georges Loinger se voit confier un autre rôle très important qui est la mise en place d'une filière clandestine pour les passages d'enfants en Suisse. Ainsi, en 1943-1944, plusieurs convois d'une vingtaine d'enfants chacun quittent Lyon deux à trois fois par mois pour Annemasse en Haute-Savoie. Là, grâce à l'entremise du maire de la ville, Jean Deffaugt, les enfants séjournent quelque temps dans un centre d'accueil. Pendant leur séjour, ils se rendent régulièrement dans une prairie qui jouxte la frontière suisse. Georges Loinger y organise des parties de jeux de ballon. Vient un moment où la balle franchit la frontière et les enfants en font de même. Une fois cette astuce éventée, Georges Loinger se fait aider par des passeurs " professionnels ». Plus de 350 enfants ont ainsi la possibilité d'être sauvés ${ }^{19}$. 


\section{L'intensification des persécutions antisémites} de la "Solution finale » en France. Pour la première fois, on assiste à l'arrestation, l'internement puis à la déportation de femmes et d'enfants. Ces rafles visent les juifs étrangers. Les natio-nalités concernées sont les Allemands, les Autrichiens, les Polonais, les Tchèques, les Russes (blancs et rouges) ainsi que les juifs devenus 
apatrides de par leur dénaturalisation par l'État français. Celui-ci a révisé les dossiers d'un grand nombre de ceux, juifs ou non, qui avaient été naturalisés après 1927. En effet, à cette époque, la France qui avait besoin de main-d'œuvre, avait mis en œuvre une politique de naturalisation relativement libérale dont beaucoup d'étrangers, juifs ou non, avaient pu bénéficier. Ceux qui ont été déchus de leur nationalité, environ 15000 personnes dont près de 7000 juifs, se retrouvent apatrides. Comme leur pays d'origine a parfois été purement et simplement rayé de la carte, par annexion ou démantèlement, comme la Pologne ou la Tchécoslovaquie, il ne leur est pas possible de récupérer leur nationalité précédente et de toute façon, dix à quinze après, ce serait bien trop tard. Ces gens privés de patrie ne sont protégés par aucun État. Il convient néanmoins de noter que le terme d'« apatride " utilisé par les historiens est quelque peu erroné car il ne figure pas dans les textes réglementaires officiels du gouvernement de Vichy. Par ailleurs, dans les tractations entre Pierre Laval et les autorités allemandes, le terme de « réfugié » ne pouvait pas non plus être utilisé. C'est pour cela que le terme d'apatride a été introduit dans l'historiographie sur la période de l'Occupation. Il rappelait l'une des expressions de la propagande antisémite de l'époque où le juif était généralement désigné comme quelqu'un n'ayant aucun lien affectif avec une quelconque patrie. Le juif était « apatride» par nature ${ }^{21}$.

La grande rafle de juillet 1942 frappe les hommes de 16 à 60 ans et les femmes de 16 à 55 ans. Les enfants de 2 à 16 ans sont néanmoins arrêtés avec leurs parents et enfermés avec eux au Vélodrome d'hiver à Paris. Sont entre autres exemptés les conjoints ou veufs de non-juifs, les femmes enceintes qui doivent accoucher prochainement et les femmes ayant un enfant de moins de 2 ans. Si un membre de la famille est exempté, les enfants de moins de 16 ans ne sont pas emmenés ${ }^{22}$.

Plus de 12884 personnes sont finalement arrêtées. Toutes le sont par la seule police française. Il y a 3031 hommes et 5802 femmes auxquelles viennent s'ajouter 4051 enfants de 2 à 16 ans dont plus de 3000 sont de nationalité française. Étant nés sur le sol français et déclarés français par leurs parents, ces enfants ont de fait la nationalité française.

Les célibataires et les couples sans enfant sont directementconduits à Drancy tandis que les familles sont transférées vers les camps de Pithiviers et Beaune-la-Rolande dans le Loiret après un transit de quelques jours au Vélodrome d'hiver. Après la déportation des adultes, les enfants de ces deux camps restent seuls, livrés à eux-mêmes, jusqu'à ce que Dannecker obtienne l'aval d'Adolf Eichmann à Berlin pour qu'ils partent également. Ils sont alors transférés au camp de Drancy d'où, mêlés à des juifs étrangers provenant de la zone sud, ils sont déportés vers Auschwitz-Birkenau entre le 17 et le 28 août. Aucun d'entre eux n'est revenu.

41 En août 1942, alors que la zone libre n'est pas encore occupée par les Allemands, le gouvernement de Vichy livre à l'occupant quelque 10000 juifs, provenant des camps d'internement ou raflés dans les grandes villes du sud de la France.

En vue de préparer la grande rafle de la zone libre, Vichy adresse aux préfets la liste des nationalités visées qui sont les mêmes que celles de la rafle parisienne. S'y ajoutent ceux qui sont entrés en France depuis le $1^{\text {er }}$ janvier 1936.

Mais, craignant certainement un nombre d'arrestations insuffisant, il supprime, le 18 août 1942, onze exemptions parmi celles qu'il avait établies deux semaines plus tôt. Seules six sont conservées. Il en résulte que les moins de 18 ans, non accompagnés, ne 
seront plus épargnés ${ }^{23}$. Cela signifie donc que la police française peut arrêter les enfants hébergés dans les homes de la zone sud car étant placés, ils ne sont pas considérés comme accompagnés. Mais, le 19 août, l'exemption concernant les moins de 16 ans est rétablie. Cette mesure n'épargne malheureusement pas ceux qui sont âgés de 17 et 18 ans. C'est ainsi qu'à la fin d'août 1942, plus de 130 adolescents de cet âge sont arrêtés alors qu'ils se trouvaient dans les maisons de l'OSE, notamment celles de la Creuse.

1942 est l'année la plus noire pour les juifs de France. Ainsi, 43 des 76 convois sont partis cette année-là emportant avec eux 6500 enfants sur les 11000 qui ont quitté la France sans retour. Deux raisons essentielles: les familles juives ont été arrêtées par surprise puisqu'avant la rafle des 16 et 17 juillet, on n'arrêtait pas les enfants. D'autre part, en 1942, les arrestations ne visaient que les juifs étrangers, lesquels étaient nombreux et très vulnérables.

\section{La difficile préparation des enfants à la vie clandestine}

Les maisons d'enfants, qui étaient jusqu'alors des refuges, deviennent de véritables pièges puisqu'elles ne sont plus à l'abri des rafles. Elles vont toutefois, à partir de septembre 1942, servir de plaques tournantes dans le processus de dissimulation des enfants. En effet, c'est là où on les prépare, quand c'est encore possible, à la vie clandestine. Leur séjour dans les maisons est donc une étape essentielle au cours de laquelle on les prépare à une nouvelle vie et surtout à une nouvelle identité. En effet, avant de les confier à des filières qui les feront passer en Suisse ou les cacheront chez des non-juifs, c'est-à-dire dans des familles d'accueil ou dans des institutions, religieuses le plus souvent, on leur assigne une fausse identité, une identité dite «aryenne ». Par " aryenne», il faut entendre "non juive». À cette occasion, on improvise des méthodes pour que les plus jeunes assimilent au mieux cette nouvelle identité et renoncent provisoirement à toute référence à leur passé. Grâce au jeu, appelé «jeu du mensonge» par les éducateurs, les enfants doivent répéter inlassablement, sans faille et surtout sans aucune hésitation, leur nouvel état-civil ${ }^{24}$. On a parfois recours à des comptines ou à des petites scènes jouées avec les enfants. Il leur faut s'approprier une nouvelle histoire familiale, une nouvelle religion, en un mot, une nouvelle vie. Il faut aussi leur apprendre à ne pas se faire remarquer par des attitudes peureuses susceptibles d'attirer les soupçons. Mais, au fur et à mesure que l'Occupation se prolonge, cette phase de préparation intense devient de moins en moins possible. Il faut alors improviser en quelques heures des méthodes pour que les enfants assimilent des consignes très strictes. L'attitude face à ces contraintes varie selon l'âge et la maturité des enfants. En général, les petits de 4 à 7 ans ont des difficultés liées à l'oubli ou à la réticence de s'affubler d'un nom qui ne leur appartient pas. Le fait même de mentir leur pose souvent un problème. D'autres ont peur qu'avec un autre nom, leurs parents ne puissent plus les reconnaître ou les retrouver. Aussi, veulent-ils rester tels qu'ils les ont quittés.

Ces enfants étant en danger de mort, l'enjeu d'alors n'est plus leur parfaite intégration mais leur survie coûte que coûte. Comme ce sont en premier lieu les adolescents étrangers qui sont menacés de déportation, il faut les affubler d'une «fausse vraie identité ", leur procurer une carte d'alimentation, leur apprendre les principales prières catholiques, etc. Généralement, c'est une assistante sociale qui prend en charge les enfants par petits groupes de 6 à 7 pour les acheminer, en train le plus souvent, vers 
un lieu sûr. Là encore, des consignes de sécurité strictes sont réitérées. En aucun cas, elles ne doivent être transgressées. Il est interdit de parler en allemand ou en yiddish, de garder sur soi tout objet lié à sa famille, comme des photos personnelles, ou au culte judaïque, comme un livre de prières, etc. Malgré toutes ces précautions, on ne peut pas tout maîtriser et il arrive qu'un enfant interprète les consignes à sa manière, volontairement ou non. Il s'agit de transposer ces enfants dans une nouvelle culture qui leur est artificielle, qui leur était étrangère.Par exemple, cette anecdote qui illustre bien l'insouciance mais aussi la méconnaissance religieuse avec laquelle il fallait compter. Un jour, dans un train, deux enfants voyageant sous de fausses identités discutent et l'un d'eux explique qu'il est triste car il n'a pas eu le temps de saluer la femme du curé avant de partir... Il s'agissait bien entendu de la femme d'un rabbin ${ }^{25}$.

47 Les rafles et les arrestations individuelles ont lieu jusqu'à la Libération, sauf dans la zone d'occupation italienne où, entre novembre 1942 et septembre 1943, les juifs sont en lieu sûr. Cette zone correspond en gros à l'ensemble des départements situés à l'est du Rhône; cependant Lyon et Marseille n'en font pas partie. Cependant, suite aux protestations des Églises et à l'aide accrue d'une partie de la population française envers les persécutés, la Police de Vichy freine progressivement son enga-gement aux côtés des Allemands, limitant ainsi leur efficacité dans le domaine de la chasse aux juifs. On peut dire que l'accueil des enfants juifs étrangers en France, dans les années trente, a été en grande partie organisé et pris en charge par la communauté juive elle-même. Quand il s'est agi de la survie de ces enfants, elle a su créer des structures clandestines capables de mettre en œuvre une vaste opération de sauvetage à l'intention des plus vulnérables, c'est-à-dire principalement ceux dont les parents étaient étrangers. La résistance juive a pu mener à bien sa mission grâce à la participation active de nombreux non-juifs qui l'ont aidée de différentes manières à cacher les enfants. 
Illustration 1. Source : Mémorial de la Shoah / CDJC - CCCLXXII-1
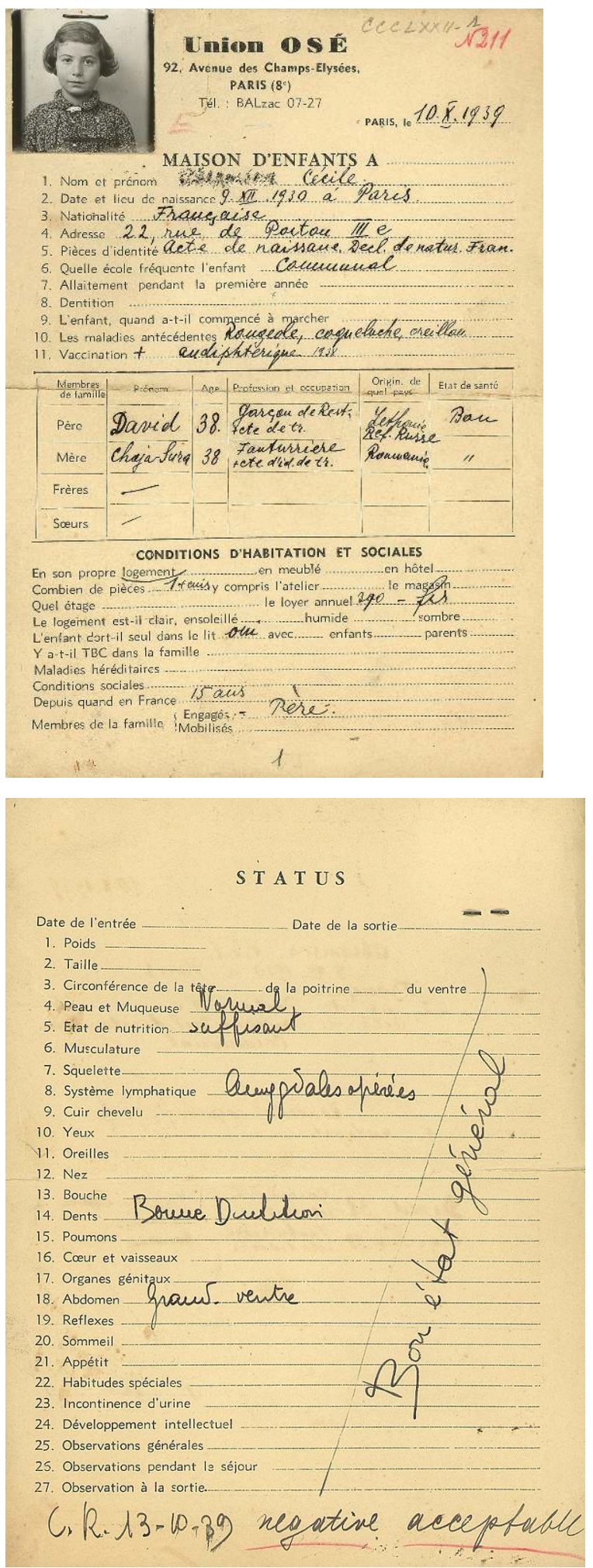
Illustration 2. Source : Mémorial de la Shoah / CDJC - CCCLXXIV-14
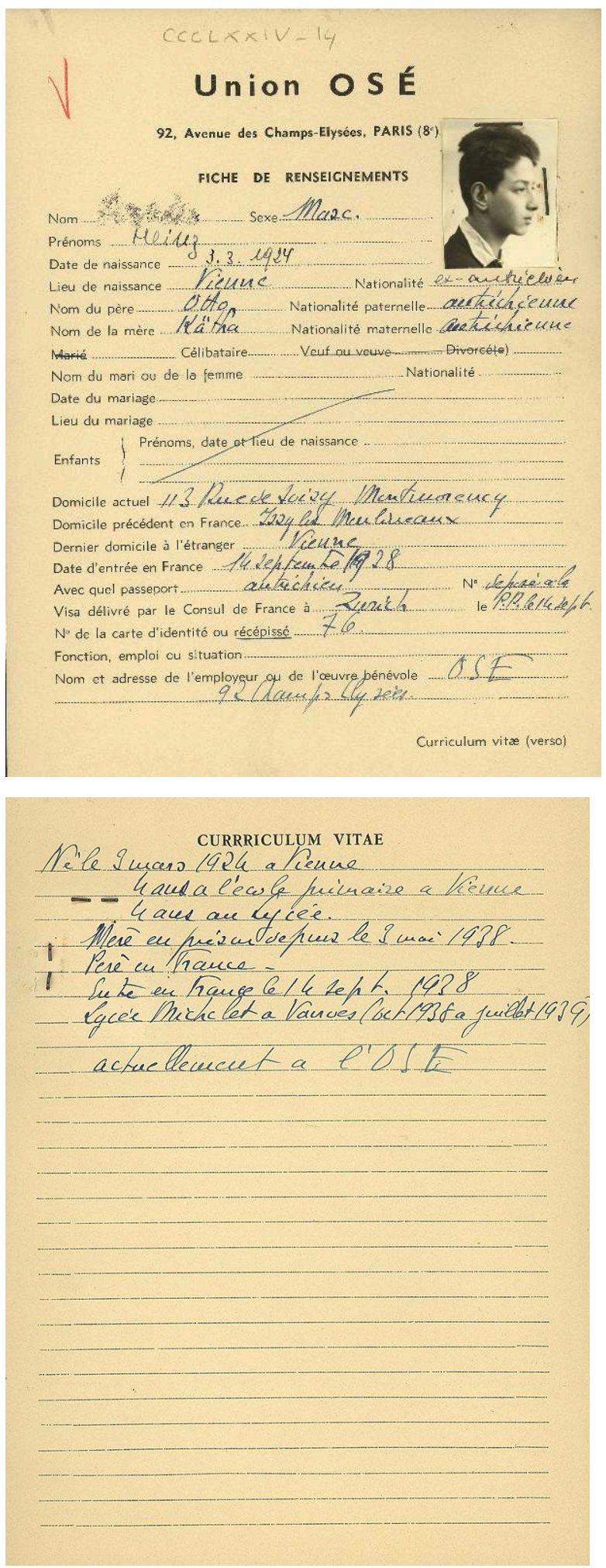


\section{NOTES}

1. Anne Grynberg, "L'accueil des réfugiés juifs d'Europe centrale en France (1933-1939) », in Les Cahiers de la Shoah, n 1,Éd. Liana Levi, 1994.

2. David H. Weinberg, Les Juifs à Paris de 1933 à 1939, Paris, Calmann-Lévy, collection Diaspora, 1974.

3. Euvre de secours aux enfants, prononcez « OSÉ ». Lors de sa création à Saint-Pétersbourg en 1912, cette organisation s'appelait Obshetsvo Zdravookranenya Yevreyiev (OZE), « Société pour la protection sanitaire des populations juives ".

4. Sabine Zeitoun, L'Euvre de secours aux enfants sous l'Occupation en France. Du légalisme à la résistance (1940-1944), préface Serge Klarsfeld, Paris, L'Harmattan, 1990.

5. Alfred et Françoise Brauner, L'accueil des enfants survivants, Paris, Groupement de recherches pratiques pour l'enfance, 1994, p. 94-95.

6. E. Mazour et O. Ratner, In fight for the health of Jewish people (50 years of OSE),Editor Dr. L. Wulman, New York, 1968, p. 33.

7. OSE Review, janvier 1937.

8. Les Chemins de liberté. Histoire des Kindertransport, Mark Jonathan Harris, 2000 (film documentaire).

9. Ernst Papanek et Edward Linn, Out the fire, New York, Ed. Morrow, 1975.

10. Der Stürmer : journal hebdomadaire allemand nazi de 1923 à 1945.

11. Bundes Deutscher Mädel (Ligue des jeunes filles allemandes) : branche féminine des Jeunesses hitlériennes, filles âgées de 14 à 18 ans. L'appartenance à ce mouvement devient obligatoire à partir de 1938 .

12. Le voyage des enfants de la Guette, film de Andrea Morgenthaler, 1990, in Les enfants de la Guette. Souvenirs et documents (1938-1945),CDJC, p. 30.

13. Alfred Brauner, Ces enfants ont vécu la guerre, Editions sociales françaises, 1946, p. 83.

14. Alfred Brauner, Ces enfants ont vécu la guerre, op. cit., p. 85.

15. Alfred et Françoise Brauner, L'accueil des enfants survivants, Groupement de Recherches pratiques pour l'Enfance, 1994, p. 92.

16. Alfred et Françoise Brauner, L'accueil des enfants survivants, op. cit., p. 103.

17. Diane Afoumado, Exil impossible ; l'errance des juifs du paquebot Saint-Louis, Paris, L'Harmattan, 2005.

18. S. Zeitoun, L'OSE sous l'Occupation en France..., op. cit.

19. Georges Loinger et Sabine Zeitoun, Les résistances juives pendant l'Occupation, Paris, Albin Michel, 2010.

20. Serge Klarsfeld, Le calendrier de la persécution des juifs en France, 1940-1944, Paris, Éd. FFDJF, 1993, p. 191-195.

21. Gérard Gobitz, Les déportations des réfugiés de zone libre de 1942, Paris, L'Harmattan, 1996, p. 268.

22. Serge Klarsfeld, Le calendrier..., op. cit., p. 272.

23. Serge Klarsfeld, Le calendrier..., op. cit., p. 389.

24. Sabine Zeitoun, L'OSE sous l'Occupation..., op. cit.

25. Sabine Zeitoun, Ces enfants qu'il fallait sauver, Paris, Albin Michel, 1989. 


\section{RÉSUMÉS}

$\mathrm{Au}$ cours des années trente, par vagues successives, des dizaines de milliers de juifs fuyant l'Allemagne, l'Autriche, la Pologne et la Tchécoslovaquie viennent se réfugier en France. Parmi eux, il y a de nombreux enfants dont certains arrivent seuls. Un organisme créé en 1938, le Comité israélite pour les enfants venant d'Allemagne et d'Europe centrale, va les prendre en charge. Il intervient par le biais de l'œEuvre de secours aux enfants (OSE), une organisation juive médico-sociale, riche d'une solide expérience. Pour secourir les nouveaux venus, elle ouvre plusieurs établissements dotés d'un personnel éducatif formé au travail social et originaire lui aussi d'Europe centrale, tout comme ses pensionnaires.

À la déclaration de guerre, en septembre 1939, Allemands et Autrichiens, considérés comme « ressortissants ennemis », sont enfermés dans des camps insalubres, avec leurs enfants. Dans ces mêmes camps du sud de la France, Vichy fait interner à partir de l'automne 1940 des milliers de familles juives étrangères. Dans les deux zones, les autres juifs sont exclus de la vie économique et mis au ban de la société par les lois françaises et les mesures allemandes. En 1941, en zone occupée, plusieurs milliers d'hommes sont arrêtés.

Les rafles de l'été 1942 marquent un tournant décisif dans le processus de la « Solution finale » en France car les arrestations et les déportations n'épargnent désormais plus les femmes et les enfants. Des œuvres, dont principalement l'OSE, s'efforcent de venir en aide à ces derniers, notamment, en les faisant sortir des camps d'internement. Issus pour la plupart de familles étrangères, ils sont regroupés dans des maisons qui avaient été ouvertes dès 1940 dans le sud de la France. Ils y reçoivent une instruction scolaire de même que, pour certains, une éducation religieuse. Les activités y sont nombreuses et variées. Le personnel éducatif, lui-même d'origine étrangère, est à même de comprendre la spécificité culturelle et linguistique de ces enfants ou adolescents.

Ces maisons, qui ne sont pas à l'abri des rafles, deviennent aussi rapidement des lieux où l'on prépare les enfants à la vie clandestine. Avant de les confier à des filières qui les cacheront chez des non-juifs (familles d'accueil et institutions religieuses ou laïques) ou les feront passer en Suisse, on leur assigne une fausse identité. Ces enfants étant en danger de mort, l'enjeu alors n'est plus leur intégration mais leur survie.

During the thirties, in successive waves, tens of thousands of Jews fleeing Germany, Austria, Poland and Czechoslovakia came to find shelter in France. Among them were a lot of children, some of them alone. The Jewish Committee for children from Germany and Eastern Europe was created in 1938 to take care of them. It operated through the medico-social Jewish organization OSE (CEuvre de secours aux enfants), which was already experienced. In order to rescue the newcomers, the OSE created several institutions for which they hired educators who had been trained in social work and who came from Eastern Europe, just like the children they would be taking care of.

When war was declared, in September 1939, German and Austrian immigrants were considered as "enemy citizens" and were incarcerated in unhealthy camps with their children. In these same camps, in the south of France, Vichy had thousands of foreign Jewish families interned from autumn 1940. In both zones, other Jews were isolated from society by French laws and German measures. In 1941 several thousand men were arrested in the occupied zone of France.

The roundups of summer 1942 represent a turning point in the process of the "Final Solution" in France, as women and children were arrested for the first time. Several organisations, particularly the OSE, strived to help the children by taking them out of internment camps. These children, who mostly came from foreign families, were gathered in homes that were first created 
in 1940 in the south of France. The educators, who were foreign themselves, were able to understand the cultural and linguistic specificity of these children and teenagers.

These homes were not safe from roundups and they quickly became a place where children were prepared for clandestine life. Before being left in the care of resistant organizations which would hide them in non-Jewish homes (host families or various religious or non-religious institutions) or smuggle them into Switzerland, they were given fake identities. Because the children were in mortal danger, the issue was no longer their social integration but their survival.

\section{INDEX}

Keywords : children, rescue, Jews, identity, roundups, Occupation, children homes, Papanek, persecution, clandestine, exclusion-foreigners, OSE, Resistance, internment camps, medical aid Mots-clés : enfants, sauvetage, Juifs, identité, rafles, Occupation, maisons d'enfants, Papanek, persécution, clandestins, exclusion, étrangers, OSE, Résistance, camps d'internement, aide médicale

\section{AUTEUR}

\section{SABINE ZEITOUN}

historienne

max.sab@wanadoo.fr 